\title{
Investigation and Analysis of Free Space Optical Link for Different Atmospheric Turbulences, Beam divergences and modulation formats
}

\author{
Gursimranjot Singh*, Er. Mandeep Singh** \\ *(Department of ECE, Punjabi university, Patiala \\ ** (Assistant Professor, Department of Electronics and communication, Punjabi University, Patiala
}

\begin{abstract}
In this work, a high speed free space optical (FSO) system is demonstrated by incorporating cost effective technique called spectrum slicing. Major achievement of the research is to use the nonlinear effect called self phase modulation as an application in spectrum slicing. A multichannel system is realized using the spectrum slices and each supports data rate of $2.5 \mathrm{Gbps}$ over $5 \mathrm{~km}$ link distance. Investigation is carried out for different performance degrading turbulences that affect the FSO channels such as fog and haze. Different beam divergences and aperture size of transmitter and receiver is also studied for NRZ and RZ. Outcomes after analyses revealed that NRZ in large antenna sizes under clear weather for minimum values of beam divergence is better to use than RZ modulation format.
\end{abstract}

Keywords: WDM, FSO, NRZ, RZ, RF

\section{INTRODUCTION}

Nowadays, Free space optics (FSO) has attracted much consideration in air communication due to numerous benefits over radio frequency $(\mathrm{RF})$ communications such as wide bandwidth, license free operation and security [2]. FSO is popular as compared to optical fibre communication, owing to more flexibility and cost-effectiveness, as well as more rapid and simpler for deployment and redeployment [3]. Presently, wavelength division multiplexed (WDM), which permits to hold several separate and autonomous optical channels, can carry Terabits per second capacities as well as it can be without difficulty incorporated with free space optical systems to significantly enhance the data speed in the system [4]. To cater the demands of bandwidth hungry services, WDM-FSO is a prominent and well competent way out for broadband transmission. However, WDM technology has several disadvantages also such as more complexity and high cost of operation. In order to overcome these limitations, a spectrum slicing technology is good alternate to WDM. Spectrum slicing having less complex operation as compared to WDM, which uses several intensity sources operational at different frequencies [5]. Also, WDM systems are wavelength selective and sensitive to routing of each wavelength to specific port. So, spectrum sliced WDM has identical advantages as wavelength division multiplexing and less complex, low cost, power efficient for future generation networks. Various physical parameters of FSO also play an important role in overall transmission.
Transmitter and receiver antenna diameters decide the reach and quality of reception of the system. Beam divergence is a major performance deteriorating factor along with atmospheric turbulences that needs to be addressed. Till now, many research works has been reported to generate spectrum slices [6-8], to boost weak signal with amplifiers [9], beam divergence size [10] scintillation noise compensation [11]. Although, reported techniques are good but either supports less data rate or more complexity. An optimal method of SS-WDM is needed for high speed FSO networks by generating high power slices and minimizing deteriorating effects.

In this paper, a high speed spectrum sliced system based on self phase modulation is demonstrated. System is analyzed for beam divergences, transmitter and receiver antenna diameters and atmospheric turbulences.

\section{SYSTEM SETUP}

In this work, a widely used simulation tool Optisystem is taken into account for the accomplishment of proposed work. In order to start the simulation, a data rate of the system is fixed to $1.56 \mathrm{Gbps}$ and a laser source in C-band is considered. A wavelength of $1552 \mathrm{~nm}$ emerges from the laser and fed to the special type of optical fiber called highly nonlinear fiber (HNLF). Due to the high power signal of laser, starts to vibrate the molecules of the fiber and introduces the nonlinear effect called self phase modulation. As a result of this, input spectra of the signal get broadened till it reaches to the output port as shown in Fig 2. A 
spectrum slicer of the frequency spacing $75 \mathrm{GHz}$ is incorporated in the system. After demux, a random data is modulated with the help of non-return to zero modulation format and MZM (Mach-zehnder modulator) in 4 WDM channels each. System specifications are represented in the Table 1.

Table 1 System parameters that are considered for proposed work

\begin{tabular}{|c|c|c|}
\hline S.No & Parameters & Values \\
\hline 1 & Wavelength & C-band $(1552.52 \mathrm{~nm})$ \\
\hline 2 & WDM channels & 4 \\
\hline 3 & $\begin{array}{l}\text { Frequency } \\
\text { spacing }\end{array}$ & $75 \mathrm{GHz}$ \\
\hline 4 & $\begin{array}{l}\text { Weather } \\
\text { conditions }\end{array}$ & Clear, Fog and Haze \\
\hline 5 & Distance & $5 \mathrm{~km}$ \\
\hline 6 & Beam divergence & $0.25-1 \mathrm{mrad}$ \\
\hline 7 & $\begin{array}{c}\text { Aperture of } \\
\text { antennas }\end{array}$ & $5-20 \mathrm{~cm}$ \\
\hline
\end{tabular}

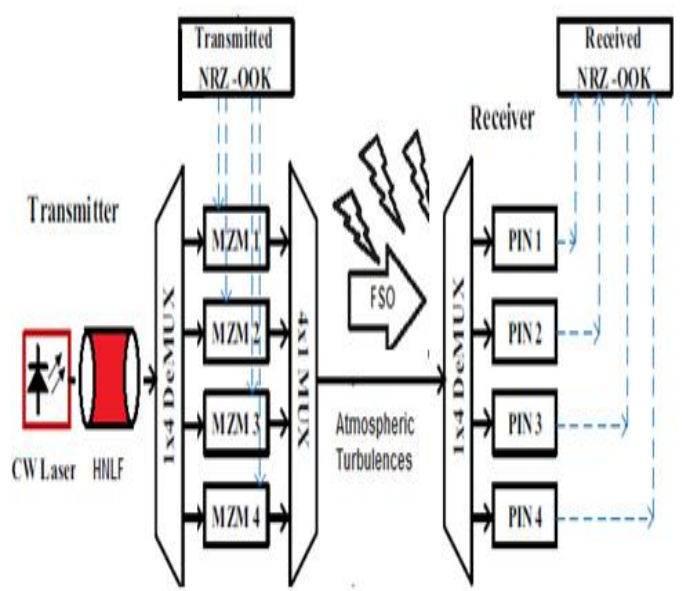

Fig 1 A 4x2.5 Gbps spectrum sliced WDM FSO system

Mach-zehnder modulator is biased at peak point and data is modulated with spectrum signal drive and NRZ. Signals are multiplexed and fed to free space optical channel that has $1 \mathrm{mrad}$ beam divergence and fixed $5 \mathrm{~cm}$ transmitter aperture, $20 \mathrm{~cm}$ receiver aperture diameter.

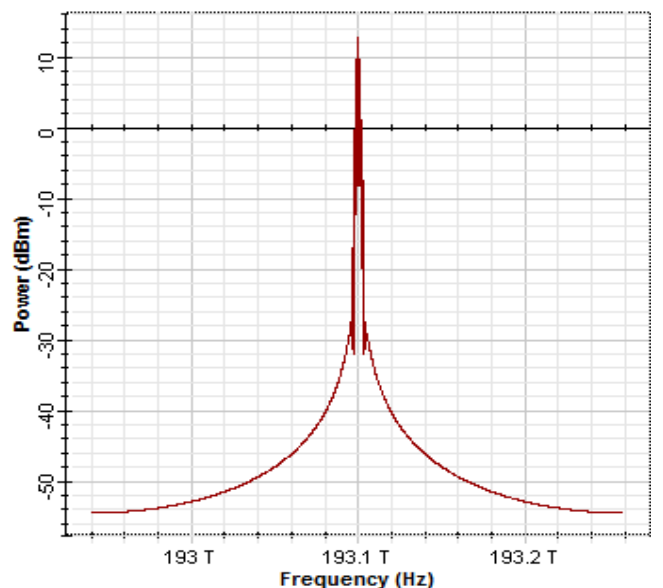

(a)

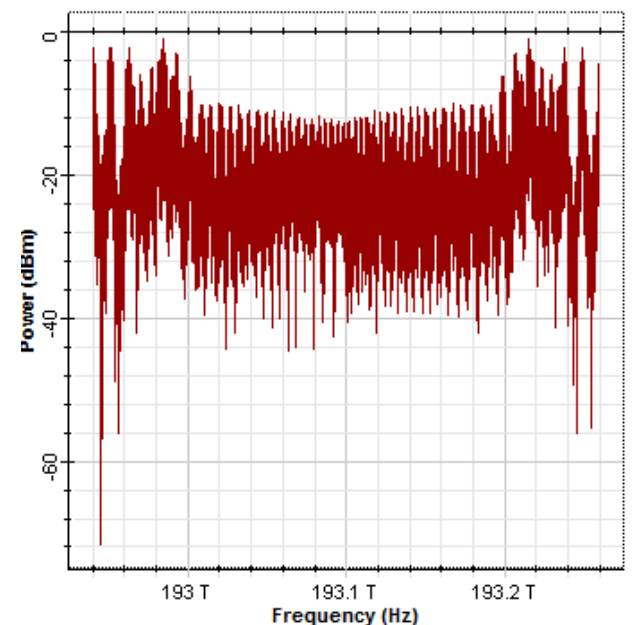

(b)

Fig 2 Depiction of the carrier spectrum for (a) without (b) with HNLF

In FSO communication, effects that are comes out to be major deteriorating issues are atmospheric instabilities such as haze and fog etc. To investigate the effects of these environmental disturbances, we have varied the attenuation of the FSO channel in the simulation and values of attenuations are taken from the reported works [12]. Attenuation values considered are such as $0.1 \mathrm{~dB} / \mathrm{km}$ for the clear weather, $4 \mathrm{~dB}$ for haze and $22 \mathrm{~dB}$ in case of fog. Moreover, second important factor to look after in the beam divergence and in this work it is varied from $0.25 \mathrm{mrad}, 0.5 \mathrm{mrad}, 0.75 \mathrm{mrad}, 0.1$ mrad. Also, effect of transmitter and receiver are also studied.

\section{RESULT AND DISCUSSION}

FSO link length is varied from $1 \mathrm{Km}$ to 5 $\mathrm{Km}$ in order to evaluate the Quality of reception in different cases of atmospheric degradations. Maximum prolonged distance is observed in clear weather conditions and minimum in case of Fog suspended particles of frozen water droplets. 
Graphical representation of different turbulences is depicted in Fig 3. It is clearly seen that Q-factor for clear weather varies from 42.57-36.24 for $1-5 \mathrm{Km}$ respectively. As in case of haze and fog is 41.5410.18 , and 28.78-0 respectively. Maximum range that can propose system cover for different turbulences is $>8 \mathrm{Km}$ for clear weather, $6 \mathrm{Km}$ for haze, $1.5 \mathrm{Km}$ for fog.

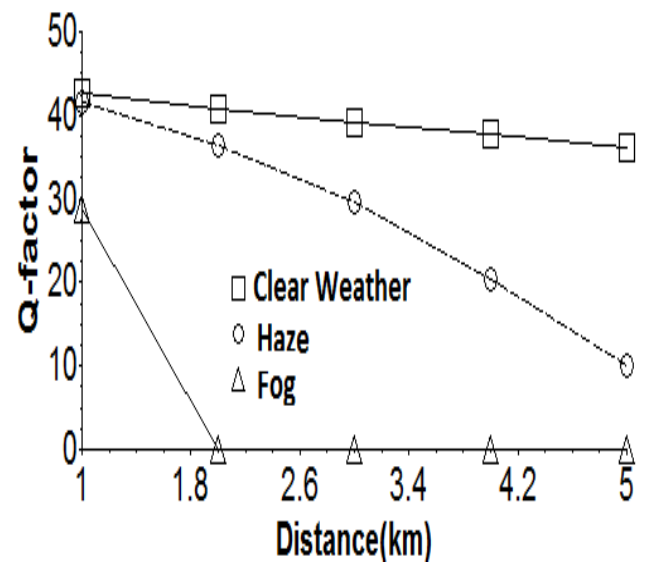

Fig 3 Performance of the system at different weather conditions

To investigate the performance of system, beam divergence angle is varied as $0.25 \mathrm{mrad}, 0.5$ mrad, $0.75 \mathrm{mrad}$ and $1 \mathrm{mrad}$. Investigation revealed that the BER tends to increase as the FSO transmitter beam gets broadened and also stated as beam divergence. Impact of beam divergence is also noteworthy; Q degrades as the beam divergence increases. As depicted in fig 4, high value of $Q$ is obtained at the least beam divergence $(0.25 \mathrm{mrad})$ and minimum BER also reported here. Since the further enhancement in beam divergence, reduce the $\mathrm{Q}$ and increase the BER. To obtain the less error in the received signal, we should use the high power and make the system with minimum beam divergence degrading effects. Also a comparison is also made for different linecoding such as NRZ and RZ at different beam divergences. Further, transmitter and receiver aperture diameters on the proposed system are also investigated in terms of $\mathrm{Q}$ factor. It is considered to be an important factor and decides the range of communication. The diameters of the Transmitter and Receiver are varied as $5 \mathrm{~cm}$, $10 \mathrm{~cm}, 15 \mathrm{~cm}$ and $20 \mathrm{~cm}$. It is evident from the results that to obtain the maximum power and $\mathrm{Q}$ at the reception, we need to increase the aperture size of receiver antenna up to $20 \mathrm{~cm}$. Fig 5 depicts the performance of the system using two modulation formats such as NRZ and RZ at different aperture diameters of the transmitter and receiver. It is evident that NRZ performs better than RZ because of more bandwidth efficiency.

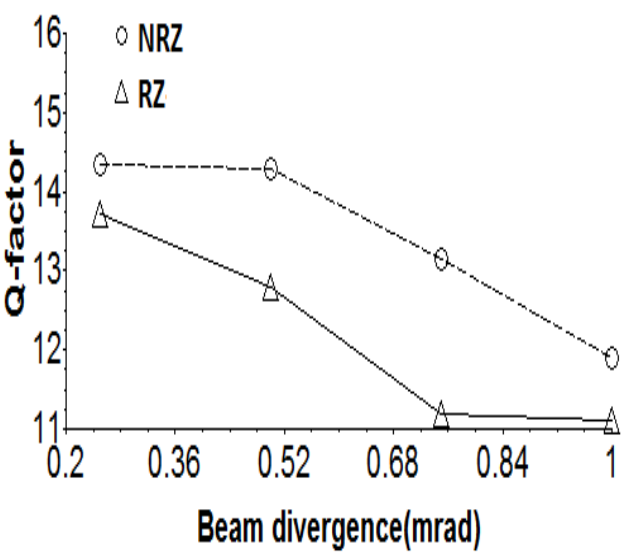

Fig 4 Effect of beam divergence on the system using NR and RZ

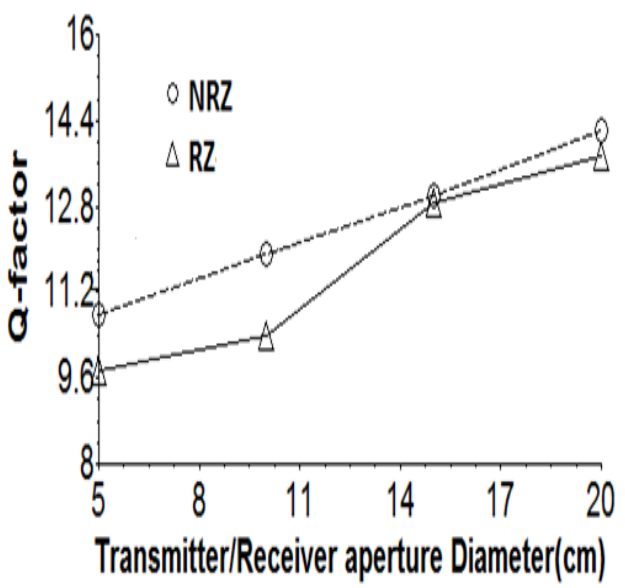

Fig 5 Effect of aperture diameters of transmitter and receiver on the system using NR and RZ

\section{CONCLUSION}

In the current investigation and evaluation, a high speed spectrum sliced free space optical (FSO) communication is demonstrated. The major achievement in this work is the use of the major degrading factor in optical fiber communication such as nonlinearity called self phase modulation. Requirement of four intensity sources is fulfilled with single laser source because of spectrum slicing. Analysis in the demonstrated system is done with NRZ, RZ, beam divergence and transmitter receiver diameters. It is reported that in spectrum sliced free space optical communication system, NRZ modulation is recommended over RZ. Beam divergence also investigated and results revealed that quality of the reception decreases on the high broadening of FSO transmitter light beam. It is evident that wide transmitter and receiver aperture augment the performance and make system able to achieve prolonged transmission. 


\section{REFERENCES}

[1] Q. Liu et al., Optical wireless communication networks for first- and lastmile broadband access [Invited], J. Opt. Netw., vol. 4, no. 12, pp. 807-828, Dec. 2005.

[2] Florence Rashidia, Jing Hea, Lin Chena, Spectrum slicing WDM for FSO communication systems under the heavy rain weather, Optics Communications, vol. 387, pp. 296-302, 2017.

[3] Sooraj Parkash, Anurag Sharma, Harsukhpreet Singh, and Harjit Pal Singh, Performance Investigation of $40 \mathrm{~GB} / \mathrm{s}$ DWDM over Free Space Optical Communication System Using RZ Modulation Format, Advances in Optical Technologies, vol. 2016.

[4] D. J. Heatley et al., Optical wireless: The story so far, IEEE Commun. Mag., vol. 36, no. 12 , pp. $72-82$, Dec. 1998.

[5] E. Ciaramella et al., 1.28 Terabit/s (32 x 40Gbit/s) WDM transmission system for free space optical communications, IEEE Journal on Selected Areas in Communications, Vol. 27, No. 9, pp. 16391645, Dec. 2009.

[6] G.J. Pendock, D.D. Sampsom, Transmission performance of high bit rate spectrum sliced WDM systems, J. Lightwave Technol., vol. 10, pp.21412148, 1996.

[7] K. Lee, D.S. Lim, M.Y. Jhon, H.C. Kim, P. Ghelfi, T. Nguyen, et al., Broadcasting in colorless WDM-PON using spectrum- sliced wavelength conversion, Opt. Fiber Technol. Vol.18, vol. 2, pp.112-116, 2012.

[8] Shin Kaneko, Jun-ichi Kani, Katsumi Iwatsuki, Akira Ohki, Mitsuru Sugo, and Shin Kamei, Scalability of Spectrum-Sliced DWDM Transmission and Its Expansion Using Forward Error Correction, Journal of Lightwave Technology vol. 24, no. 3, pp. 1295-1301, 2006.

[9] Amrutha Sara Koshy, Jaini Sara Babu, Impact of Erbium Doped Fiber Amplifier on WDM-FSO System under Rain Attenuations, Ijareeie, vol. 5, no. 2, pp. 867-872, 2016.

[10] Hemani Kaushal, Georges Kaddoum, Virander Kumar Jain, Subrat Kar, Experimental investigation of optimum beam size for FSO uplink, Optics communications, vol. 400, pp. 106-114, 2017.

[11] Mohammad Abtahi, Leslie A. Rusch, Mitigating of scintillation noise in FSO communication links using saturated optical amplifiers, Proceeding MILCOM'06 Proceedings of the 2006 IEEE conference on Military communications, pp. 31813185, 2006.

[12] Kim, B. McArthur, and E. Korevaar, Comparison of laser beam propagation at $785 \mathrm{~nm}$ and $1550 \mathrm{~nm}$ in fog and haze for optical wireless communications, Proc. SPIE, vol. 4214, pp. 26-37, Optical Wireless Communications III, edited by Eric J. Korevaar (2000).

\footnotetext{
International Journal of Engineering Research and Applications (IJERA) is UGC approved Journal with Sl. No. 4525, Journal no. 47088.

Gursimranjot Singh. "Investigation and Analysis of Free Space Optical Link for Different Atmospheric Turbulences, Beam divergences and modulation formats." International Journal of Engineering Research and Applications (IJERA) 7.7 (2017): 22-25.
} 